\title{
Computing Histograms of Local Variables for Real-Time Monitoring using Aggregation Trees
}

\author{
Dan Jurca and Rolf Stadler \\ Royal Institute of Technology (KTH) \\ Laboratory for Communication Networks LCN \\ SE-100 44 Stockholm, Sweden \\ Email: \{danjurca,stadler\}@kth.se
}

\begin{abstract}
In this paper we present a protocol for the continuous monitoring of a local network state variable. Our aim is to provide a management station with the value distribution of the local variables across the network, by means of partial histogram aggregation, with minimum protocol overhead. Our protocol is decentralized and asynchronous to achieve robustness and scalability, and it executes on an overlay interconnecting management processes in network devices. On this overlay, the protocol maintains a spanning tree and updates the histogram of the network state variables through incremental aggregation. The protocol allows to control the trade-off between protocol overhead and a global accuracy objective. This functionality is implemented by a dynamic configuration of local error filters that control whether an update is sent towards the management station or not. We evaluate our protocol by means of simulations. Our results demonstrate the controllability of our method in a wide selection of scenarios, and the scalability of our protocol for large-scale networks.
\end{abstract}

\section{INTRODUCTION}

The ability to provide continuous estimates of management variables is vital for many management tasks, including network supervision, quality assurance, and proactive fault management. Often, management variables that are monitored in these tasks are aggregates that are computed from local device variables across the network. Example aggregates are the total number of VoIP flows, the maximum link utilization, the histogram of the current load across routers, or the distribution of the router out-degree in a network domain.

While it is often crucial to know how accurate such estimates are, network management solutions deployed today usually provide only qualitative control of the accuracy and do not support the setting of an accuracy objective. Engineering continuous monitoring solutions for network management involves addressing the fundamental trade-off between accurate estimation of a variable and the management overhead in terms of traffic and processing load [1], [2].

Distributed solutions for the aggregation of local variables usually push the aggregation function inside the network and aggregate the partial result in a decentralized way. Such solutions prove to be very efficient and exhibit good load balancing properties, along with increased robustness and

This work has been part of 4WARD, a 7th Framework ISP project funded by the EC. It has further been supported by the ACCESS Linnaeus Center at KTH. resilience to network dynamics [3]. However, they are individually tailored for the specific envisioned functionality and cannot be used in case the management station switches the objective function. E.g., a single instantiation of a distributed protocol designed for aggregating SUM cannot provide an answer for a MAX or MIN query at the management station, hence a new instantiation of a different protocol is required. With this respect, the design of a distributed protocol that provides the management station with an accurate estimate of the histogram of the monitored local variable represents a worthy goal. Based on the estimate obtained through a single protocol instantiation, the management station has the flexibility of applying locally any desired statistical function, either sequentially, or in parallel, e.g., SUM, MIN and MAX queries can be answered in the same time only through local manipulation of the aggregate histogram.

In this paper, we address the problem of continuous monitoring of a local variable's histogram with accuracy objectives for large-scale network environments. Our goal is to design an efficient aggregation protocol that allows us to control the trade-off between the accuracy of the estimation and the protocol overhead. Our protocol continuously computes the distribution of the values of a monitored local variable through histogram aggregation by (i) creating and maintaining a selfstabilizing spanning tree and (ii) incrementally aggregating the variable's histogram along the tree (Figure 1). It is push-based in the sense that changes in the partial distribution of the monitored variable values are sent towards the management station along the aggregation tree. The protocol controls the management overhead by filtering updates that are sent from nodes to the management station, by allowing for local errors between the last sent updates and the present network status.

The local error filters periodically adapt to the dynamics of the monitored variables and the network environment, keeping in mind a global error objective for the monitoring process. All operations in our protocol, including computing the partial histogram of the variable and the configuration of the local error shares, are executed in a decentralized and asynchronous fashion to ensure robustness and achieve scalability. While designing our protocol we keep in mind the goals of (i) controllable accuracy, as a trade-off between aggregation accuracy and protocol overhead, (ii) dynamic adaptation to changes in the network topology, or changes in the evolution of local variables, (iii) controllability in terms of 


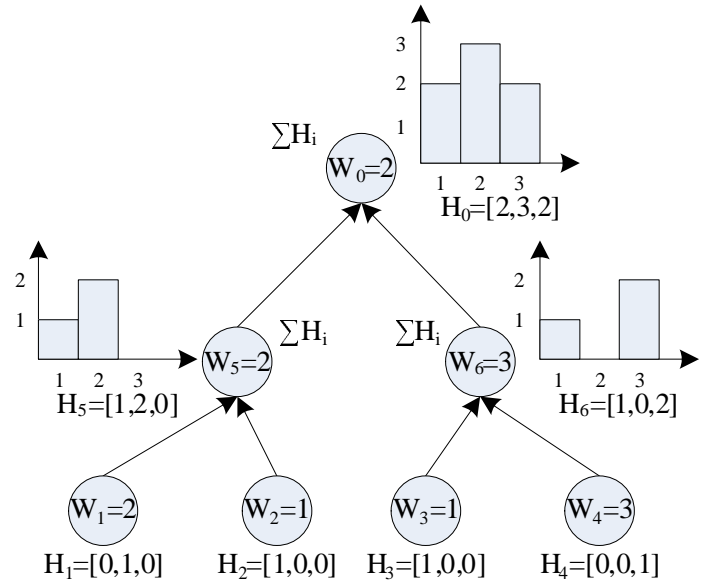

Fig. 1. Histogram Aggregation on Trees: local values $w_{i}(t) \in S$, where the value interval $S=\{1,2,3\}$. The aggregation vectors $H_{i}$ represent the partial aggregated histogram of the local values $w_{j}$ of nodes $j$ belonging to subtree $T_{i}$. Intermediate nodes in the tree aggregate the received vectors $H_{i}$ through element-wise sum.

real-time changing the accuracy objective, or protocol response to system changes, and (iv) scalability, as we design our protocol for large distributed systems.

Previous work on monitoring local network variables using aggregation trees is presented in [1] and [3]. The authors propose aggregation protocols with accuracy guarantees that function for a given aggregation function. Other works that explore the trade-off between aggregation accuracy and incurred overhead are presented in [4], [5], [6], [7]. While keeping the same principles in mind, our work is more general, as by histogram aggregation we allow the management station the flexibility in applying any aggregation function (or combination of functions) on the aggregated data. Metrics and algorithms for the dynamic maintenance of histograms or quantiles over large data sets are presented in the works described in [8], [9], [10], [11], [12], [13]. While we rely on these works for our metric definition and histogram formalization, the scope of our work is different, as we tackle the problem of efficient histogram aggregation over distributed aggregation trees. Probably the closest work to our proposed protocol is presented in [2]. The authors analyze the problem of approximate quantile tracking with accuracy objectives in management systems. They also sketch the idea of histogram aggregation in hierarchical systems using aggregation trees. However, they do not propose or analyze any complete protocol solution for this problem. With this respect, we differentiate our work by detailing a full-fledged mechanism for histogram aggregation over hierarchical trees, along with our analysis and experimental results in various network settings.

The novelty and contributions of our work are three-fold:

- To the best of our knowledge, this work is the first to present a full-fledged solution for the problem of distributed histogram aggregation with accuracy objectives using aggregation trees. Our solution is based on a formal optimization framework, and contains greedy error filter allocation algorithms;
- We formalize the trade-off between protocol overhead and accuracy objective as a global optimization problem, and we offer a full analysis based on a simple stochastic model for the evolution of the local monitored variables;

- We demonstrate the performance of our proposed solution through simulations, and we emphasize its controllability and scalability in various network scenarios.

The paper is organized as follows: in Section II we introduce our system model and the notations used throughout the paper. The global optimization problem is presented in Section IV, while Section $\mathrm{V}$ attempts its full mathematical modelling in a centralized architecture. We present our distributed heuristic algorithms in Section VI and analyze their performance in Section VII. Finally we discuss our contributions and future work in Section VIII, and we conclude our paper in Section IX.

\section{System Model AND Notations}

\section{A. System Architecture}

We assume a distributed management architecture, where each network device participates in the monitoring task by running a management process. These management processes communicate via a management overlay network for the purpose of monitoring. We refer to this overlay as the network graph $G(t)=(V(t), E(t))$. A node $n \in V(t)$ represents a management process.

We assume that the network graph contains $N$ management processes, and is organized as an aggregation tree $T_{0}$, rooted at the monitoring process 0 . Let $T_{n}$ be the subtree rooted at process $n \leq N$, and let each process $n$ run one leaf node and one aggregating node of this tree.

Each leaf $n$ has an associated local variable $w_{n}(t)$ which is an integer-valued quantity. The term local variable is used to represent a local state variable or device counter that is being subjected to monitoring. Local variables are updated asynchronously with a given sampling rate. Without loss of generality, assume that $w_{n}(t) \in S=\left\{s_{1}, \ldots, s_{m}\right\}$, where $S$ is the set of all possible values that can be taken by $w_{n}(t)$, $\forall n \leq N, \forall t$. Finally, let $\mathcal{P}$ be the generic stochastic process associated with the evolution of the local variables over time. $\mathcal{P}$ may refer to a similar stochastic process characterizing the change of all local variables, or could encompass a different process for each local variable.

\section{B. Histogram Definitions}

Let $H_{n}(t)$ be the aggregation vector representing the histogram of all local values belonging to nodes in subtree $T_{n}$. If $H_{n}^{k}(t)$ denotes the number of local variables with value $w_{j}(t)=s_{k}, j \in T_{n}$, then $H_{n}^{k}(t)=\sum_{j \in T_{n}} c_{j, k}(t)$, where:

$$
c_{j, k}(t)= \begin{cases}1 & \text { if } w_{j}(t)=s_{k} \\ 0 & \text { otherwise }\end{cases}
$$

Then we can compute the aggregation vector $H_{n}(t)=$ $\left[H_{n}^{1}(t), \ldots, H_{n}^{m}(t)\right]$, with $m=|S|$. Observe that the aggregation vector of a leaf node has a value of 0 on all vector positions, except on the position corresponding to its local variable, where it has a value of 1 . 
We define the distance between two aggregation vectors of the same length $m, H_{i}$ and $H_{j}$, as the total number of differences in the aggregated local values:

$$
D\left[H_{i}, H_{j}\right]=\sum_{k=1}^{m}\left|H_{i}^{k}-H_{j}^{k}\right| .
$$

At the same time we define the $y$-distance between the aggregation vectors as $D_{y}$ (e.g., $D\left[H_{i}, H_{j}\right]=y$ ), and $P_{y}=$ $\operatorname{Prob}\left(D\left[H_{i}, H_{j}\right]=y\right)$ as the corresponding probability of having a distance $D_{y}$ between two aggregation vectors. Notice that as long as the number of aggregated local values stays constant in time, $P_{y}=0$, for all odd numbers $y$, i.e., a single variable having different values in the two histograms leads to a distance $D_{2}$ between the histograms. Similarly, if $d$ is the number of variables aggregated in histograms $H_{i}$ and $H_{j}$, the maximum distance between the histograms when none of the $d$ values overlap is $D_{2 d}$, or $P_{y}=0, \forall y>2 d$. $P_{y}$ depends on the stochastic process $\mathcal{P}$ characterizing the change of local variable values in time.

Let $\epsilon(t)$ be the error at the management station (or root process 0 ), expressed as the distance between the aggregated vector $H_{0}(t)$ available at the root as a result of the aggregation protocol, and the real histogram of all local values in the network at time $t$. Finally we define $\epsilon_{M}$ as the maximum tolerated error by our monitoring system, leading to a minimum accuracy objective inversely proportional to $\epsilon_{M}$. $\epsilon_{M}$ influences the overhead of the aggregation protocol, as a large tolerated error allows for less frequent vector updates, hence reduced protocol overhead, while a smaller tolerated error imposes more frequent protocol updates, as local values change in time.

\section{Aggregation Protocol Notations}

The aggregation protocol sends message updates from children processes to their parents in order to aggregate the histogram of the local values at each level in the tree rooted at the management station (Figure 1). An update message sent by node $n$ contains the aggregation vector $H_{n}(t)$ available at node $n$ at time $t$. A parent process $n$ having the set of child processes $\gamma(n)$ (including its own leaf process) aggregates the received update messages and computes the latest histogram of its subtree:

$$
H_{n}(t)=\sum_{j \in \gamma(n)} H_{j}(t)
$$

Here, by $H_{j}(t)$ we denote the most recent aggregation vector available at parent node $n$ through the last message update received from child node $j$. The sum represents the element-wise sum of the elements $H_{j}^{k}$.

The update rate of child process $j \in \gamma(n)$ is denoted by $R_{j}^{\text {out }}(t)$ and is computed as the average number of update messages sent towards parent $n$ in a time window. $R_{j}^{\text {out }}(t)$ is determined by the change in monitored values compared to the last update sent, and by the maximum allowed error between updates, $\epsilon_{j}(t)$ allocated to process $j . \epsilon_{j}(t)$ should be allocated by the aggregation protocol for each individual process $j$ in the aggregation tree, as a share of the total tolerated error
$\epsilon_{M}$. At the same time, the rate of incoming updates at parent node $n$ is computed as the sum of incoming update rates: $R_{n}^{i n}(t)=\sum_{j \in \gamma(n)} R_{j}^{\text {out }}(t)$. Finally, the overhead at node $n$ is computed as: $\Omega_{n}(t)=R_{n}^{\text {in }}(t)+R_{n}^{\text {out }}(t)$.

We observe that $\Omega_{n}(t)$ is a function of the stochastic process $\mathcal{P}$ modelling the changes in local values at all processes $j$ belonging to subtree $T_{n}$ (including process $n$ ), and their associated error shares $\epsilon_{j}(t)$, e.g., $\Omega_{n}(t)=\Omega_{n}\left(t, \mathcal{P}, \epsilon_{j}(t)\right)$, $\forall j \in T_{n}$. While $\Omega_{n}$ characterizes only the number of messages exchanged by our protocol (and not the total bit-rate required), it is a good indicator for the total protocol overhead as long as the update message size is kept constant. This is in fact the case, as long as the size of the update vector is constant at all processes $n$, i.e., the cardinality of the value set $|S|=c t$ throughout the runtime of the system, at all processes.

\section{Protocol OVERVIEW}

Our objective is to engineer a protocol on the network graph that provides a management station with a continuous estimate of the histogram of the local values, for a given accuracy objective. The protocol should execute with minimal overhead, expressed as number of updates processed per second in the network. The accuracy is expressed as the maximum error of the estimate over time.

The design objectives of our protocol are four-fold:

- dynamic adaptation to changes: the algorithm should adapt in real-time either to changes in the network topology, and to changes in the local variables or the evolution of such changes;

- controllability: the response of our algorithm to system changes should be controlled in real-time with the help of different control parameters as later presented in this paper;

- scalability: we design our protocol for large, dynamic systems. Its performance should scale with the increase of system size, and should cope with system variability;

- controllable overhead-accuracy trade-off: by considering the accuracy objective as a control parameter, the overhead of our protocol should vary as a function of the required performance in terms of aggregate accuracy.

We call our presented protocol for histogram aggregation as HistAgg. HistAgg is based on GAP (Generic Aggregation Protocol), developed in our earlier work [14]. GAP is an asynchronous distributed protocol that builds and maintains a BSF spanning tree on an overlay network, in a similar way as the algorithm that underlies the 802.1d Spanning Tree Protocol [15].

In GAP each node holds information about its children in the BSF tree, in order to compute the partial aggregate, i.e., the aggregate value of the local management variables from all nodes of the subtree where this node is the root. GAP is event-driven in the sense that messages are exchanged as results of events, such as the detection of a new neighbor on the overlay, the failure of a neighbor, an aggregate update or a change in the local management variable.

A problem experienced in GAP is that it can cause a high load on the root node or on nodes close to the root, specifically 
in large networks. In order to reduce this overhead, one can either apply a rate limitation scheme, which imposes an upper bound on message rates on each link, or one can introduce a filter scheme, whereby a node drops updates when only small variations of its partial aggregate occur [1]. In both cases, the protocol overhead is reduced at the cost of introducing an error in the aggregate estimation process.

For HistAgg, we choose an error filter scheme that forwards a message update from a node $n$ to its parent only when its current partial aggregate differs more than a given error threshold $\epsilon_{n}$ from the last reported aggregate. The filter scheme is built as an extension of the GAP protocol. The functionality of GAP that is inherited by Hist Agg is (i) creating and maintaining the aggregation tree (and specifically handling node arrivals, departures and failures) and (ii) performing the aggregation function on the tree using generic functions and data structures related to maintaining partial aggregates at parent nodes at different levels in the tree.

In the following sections we express our protocol objectives in the form of a global optimization problem and offer its full formalization. Afterwards we provide heuristic algorithms which offer greedy solutions in distributed and asynchronous network settings.

\section{Optimization PRoBlem}

Under the given system setup and notation, we want to derive an update protocol which computes in real time an estimate of the histogram of the monitored local values inside the network. We are interested in minimizing the total overhead of such protocol, while still fulfilling the accuracy objective imposed on our setup. Formally, we denote as $\epsilon \overrightarrow{(t)}=$ $\left[\epsilon_{0}(t), \ldots, \epsilon_{N}(t)\right]$ the vector of all error shares distributed by our algorithm to all processes in the management system at time $t$. We want to compute:

$$
\left.\epsilon \overrightarrow{(t)}{ }^{*}=\underset{\forall(\epsilon(t))}{\arg \min } \sum_{n=0}^{N} \Omega_{n}\left(t, \mathcal{P}, \epsilon_{j}(t)\right)\right)
$$

such that the total error constraint is satisfied at all times:

$$
\epsilon(t) \leq \epsilon_{M}, \forall t
$$

Hence, the optimization problem translates into finding the optimal split of the total system tolerated error $\epsilon_{M}$ into local shares $\epsilon_{n}$.

Notice that for a given fixed stochastic process $\mathcal{P}$, the total protocol overhead depends on all individual update rates at each process $n$ in the tree, which in turn depends on the allocated local shares of tolerated errors $\epsilon_{n}$.

We attempt a full formalization of the optimization problem under some strict assumptions and for a simple stochastic process for the local value evolution in time in the next section.

\section{ANALYSIS}

In this section we analyze the optimization problem for a simple update process $\mathcal{P}$ for all local variables $w_{n}(t)$. For the sake of simplicity, we assume that the network is synchronized, and that all local values change periodically with the same period $\tau$. We assume that at each time step, each process updates its local variable to a new value from $S$ according to a uniform distribution. Hence, this memoryless process yields at each time step, or current time $t_{c}$, local values $w_{n}\left(t_{c}\right)=s_{k}, \forall n \leq N, \forall k \leq m$, where $m=|S|$, with probability:

$$
P\left(w_{n}\left(t_{c}\right)=s_{k}\right)=\frac{1}{m}, \forall t_{c}, \forall n, \forall k
$$

Notice that updates of value $w_{n}$ do not depend on the specific time step in the runtime of the system. Hence, in our analysis we simplify our notations whenever possible, and do not refer to time anymore.

\section{A. Leaf Processes}

Leaf processes $n$ do not receive any incoming messages, as there is no subtree $T_{n}$ rooted at $n$. Hence $R_{n}^{i n}\left(t_{c}\right)=0, \forall t$. We assume that each leaf process $n$ sends a message update to its parent as soon as the local value $w_{n}$ changes value compared to the last sent update, e.g., $w_{n}\left(t_{c}\right) \neq w_{n}\left(t_{u}\right)$, or $\epsilon_{n}=0$. We denote the time of the last sent update as $t_{u}$. According to $\mathcal{P}$, this happens with probability $P\left(w_{n}\left(t_{c}\right) \neq w_{n}\left(t_{u}\right)\right)=1-\frac{1}{m}$. Hence the outgoing rate of message updates of a leaf process normalized with the time period is:

$$
R_{n}^{\text {out }}=\frac{m-1}{m},
$$

and the associated protocol overhead with leaf process $n$ is $\Omega_{n}=R_{n}^{\text {out }}$.

\section{B. Aggregating Processes}

An aggregating process $n$ is the root node of the subtree $T_{n}$, and receives update messages from all its children $j \in \gamma_{n}$. The incoming rate perceived by process $n$ is $R_{n}^{i n}=\sum_{j \in \gamma_{n}} R_{j}^{\text {out }}$, where $R_{j}^{\text {out }}$ can be the outgoing message rate of a leaf process or another aggregating process.

$R_{j}^{\text {out }}$ of an aggregating process $j$ can be computed as the probability of a distance larger than $\epsilon_{j}$ between the last sent update and the current aggregation vector. For notation simplification we denote as $D(j)=D\left[H_{j}\left(t_{c}\right), H_{j}\left(t_{u}\right)\right]$. Under our model $\mathcal{P}$, this probability is invariant in time, hence we have:

$$
R_{j}^{\text {out }}=\operatorname{Prob}\left(D(j)>\epsilon_{j}\right) .
$$

Let $P^{1}=\operatorname{Prob}\left(D(n)>\epsilon_{n}\right)$. Then we can compute:

$$
P^{1}=\sum_{y=\epsilon_{n}+1}^{2 d(n)} P_{y}(n),
$$

where $P_{y}(n)$ is the probability of a $y$-distance between two aggregating vectors $H_{n}\left(t_{c}\right)$ and $H_{n}\left(t_{u}\right)$ as defined in the previous sections, and $d(n)$ is the size of the subtree $T_{n}$ rooted at process $n$.

$P_{y}(n)$ is computed at process $n$ as the distance between the last update vector sent up on the tree and the newly computed 
aggregated vector, as summed from message updates received from children. It depends on the individual distances reported in each of the update vectors received from children, taking into account that in the final aggregated vector $H_{n}$ distances from different children vectors $H_{j}$ might cancel each other out.

If $c(n)=\left|\gamma_{n}\right|$ is the number of direct children of process $n$ in tree $T_{n}$, then $P_{y}$ can be recursively computed at process $n$ as:

$$
P_{y}(n)=\sum_{a_{1}=1}^{2 d(1)} \ldots \sum_{a_{c(n)}=1}^{2 d(c(n))}\left[P_{y}^{2}(n) \prod_{i=1}^{c(n)} P_{a_{i}}(i)\right]
$$

where $P_{y}^{2}(n)$ is the probability that the distance between the last updated vector $H_{n}\left(t_{u}\right)$ and the newly computed vector $H_{n}\left(t_{c}\right)$ is equal to $y$ (e.g., $D(n)=y$ ) given that the distance between the vectors from any child $j$ is $a_{j}$ (e.g., $D(j)=a_{j}$ ):

$$
P_{y}^{2}(n)=\operatorname{Prob}\left(D(n)=y \mid D(j)=a_{j}, \forall j \in \gamma_{n}\right)
$$

$P_{y}^{2}$ can be numerically computed under the assumption of $\mathcal{P}$ knowing the size of the subtree $d(j)$ rooted at any child process $j$ of process $n$, starting from the binomial distribution of the probability of a distance $D_{k}$ out of a maximum possible distance $D_{2 d}: P_{2 d}(k)=\left(\begin{array}{c}2 d \\ k\end{array}\right) p^{k}\left(1-p^{2 d-k}\right)$, with $p=\frac{m-1}{m}$.

After computing $R_{n}^{\text {out }}$, a centralized algorithm that knows the set of children $\gamma_{n}$ of any process $n$ in the aggregation tree $T_{0}$ can compute the optimal vector allocation $\overrightarrow{\epsilon(t)}{ }^{*}$, and hence solve the optimization problem.

In the next section we present our heuristic algorithm that computes an approximation of the optimal error share allocation for all processes in the tree in a distributed way, with no assumption on full topology knowledge or stochastic process $\mathcal{P}$.

\section{Distributed Solution}

This section details our heuristic solution for the distributed computation of local error shares $\epsilon_{n}$ which influence the overhead of HistAgg under the total error objective $\epsilon_{M}$. First we approximate the global optimization problem with local optimization problems that can be solved in a distributed way at all parent nodes in the tree. Then we provide a fast heuristic algorithm which offers a solution for the local problem.

\section{A. Distributed Optimization Problem}

Let process $n$ have an allocated error budget $\epsilon_{n}$ that must be distributed to all child processes in aggregation tree $T_{n}$. As before, let $c(n)=\left|\gamma_{n}\right|$ be the total number of direct children of process $n$ and let $d(n)$ be the size of $T_{n}$. We also denote as $\epsilon(\vec{n})=\left(\left\{\epsilon_{j} \mid \forall j \in \gamma_{n}\right\}\right)$ the allocation of error shares to all child processes. We want to compute:

$$
\epsilon(\vec{n})^{*}=\underset{\forall \epsilon(\vec{n})}{\arg \min } \Omega_{n}(\epsilon(\vec{n}))
$$

under the constraint: $\sum_{j \in \gamma_{n}} \epsilon_{j} \leq \epsilon_{n}$.
With a full knowledge of the behavior of partial aggregates from children, a full-search algorithm can yield the optimal vector $\epsilon(\vec{n})^{*}$, and hence solve the optimization problem. However, monitoring and estimating even on short time windows the evolution of the partial children aggregates requires a high volume of information exchange and storage [3], which in the end might defeat the purpose of our solution. Hence, in the rest of this paper we concentrate on a solution based on a faster greedy algorithm which scales even for large network systems.

\section{B. Protocol Initialization}

HistAgg initializes by constructing a spanning tree on the management overlay in the same way as GAP does [14]. On all nodes of the aggregating tree the error shares are initialized to zero, so that all changes in the partially aggregated histograms are reported up the aggregation tree to the root node. During a warm-up period, each parent node $n$ monitors the incoming message rate from all it children and finds the child node that loads the network most, by sending the largest number of updates. After the warmup period the accuracy objective $\epsilon_{M}$ is set at the root node and the protocol starts running in a distributed way.

\section{Overhead Estimation}

Each parent node estimates the overhead for each of its children, in order to identify at all times the child that induces the largest protocol overhead. We achieve this by maintaining at the parent node, for each of its children, a circular buffer which records the rate of child updates during a given monitoring window.

Let $R_{j}^{\text {out }}(t)$ be the rate of updates send by child node $j$ (e.g., the number of message updates sent by node $j$ towards its parent in a time unit), and let $T$ be the size of the monitoring window, measured in time units. Then, at any time $\tau$ the average rate estimate for child $j$ is: $R_{j}^{\text {out }}=\frac{1}{T} \sum_{t=\tau-T}^{\tau} R_{j}^{o u t}(t)$, and the estimate of the total incoming rate as seen by parent $n$ is $\hat{R_{n}^{i n}}=\sum_{j \in \gamma_{n}} R_{j}^{\text {out }}$.

The choice of the window size influences the performance of our algorithm. On the one hand, the variance of our estimator is inversely proportional to the window size. On the other hand, a short window size makes the system more adaptive.

\section{Heuristic $\epsilon(\vec{n})$ Allocation Algorithm}

We present a polynomial-time algorithm as an efficient heuristic to find a solution vector $\epsilon(\vec{n})$ to Eq. (4). The computation is based on the step-wise optimization of our allocation of $\epsilon_{j}$. Our algorithm uses the child overhead estimation as presented above, in order to compute step-wise the child that induces the largest protocol overhead.

Starting from an initial error share allocation $\epsilon(\vec{n})$, at each iteration, the algorithm increases by $\Delta \epsilon$ the error share of the child that has the highest $R_{j}^{\hat{o u t}}$, in an attempt to reduce 
the protocol overhead as seen by parent $n$. The iterations are performed with periodicity $t_{i}$, during which the parent monitors the effect of allocating the extra share $\Delta \epsilon$ to node $j$ and computes a crude estimate of its new $R_{j}^{\hat{o u t}}$.

The algorithm terminates when the error budget $\epsilon_{n}$ is depleted, or when all child nodes become passive, i.e., their error share is large enough to prevent all aggregation updates towards the parent.

The pseudo-code of the algorithm is presented in Algorithm 1:

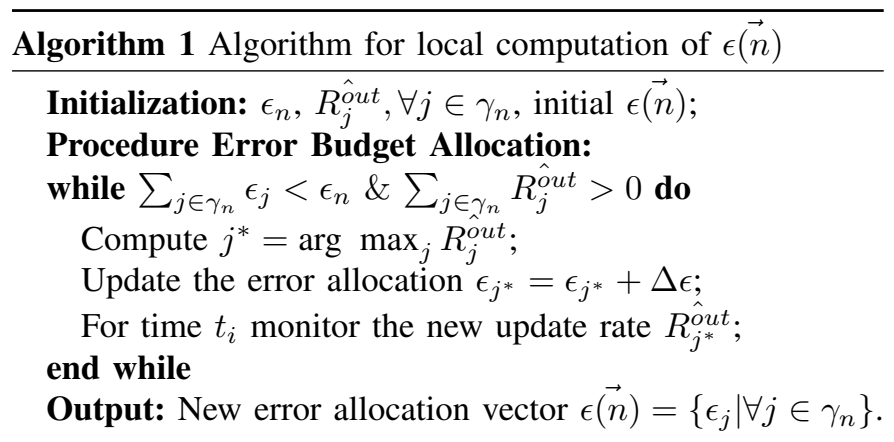

Notice that $R_{j}^{\hat{o u t}}$ can be monitored and roughly estimated on a time interval $t_{i}$ which is proportional to the sampling interval of the monitored local weights.

Algorithm 1 iteratively distributes the error budget $\epsilon_{n}$ to child processes, in a manner that attempts the minimization of the sum of the incoming update rates from children. At each step, the algorithm increases the error budget of the child process that induces the highest protocol overhead. Recall that at system setup, when the algorithm is run for the first time, the initial error allocation vector is zero, e.g., $\epsilon(\vec{n})=0$.

At each iteration in the while loop, the algorithm searches the child $j^{*}$ with the maximum outgoing rate. Without any other computational or memory requirements during run-time, this operation requires $\left|\gamma_{n}\right|$ separate computations. The number of iterations is limited by the total error budget $\epsilon_{n}$. If $\epsilon_{n}$ is expressed as an integer number corresponding to the maximum tolerated distance between two aggregating vectors, and the minimum error share $\Delta \epsilon$ represents the constant minimumincrease step used by the algorithm, then the number of algorithm iterations is limited by $\frac{\epsilon_{n}}{\Delta \epsilon}$. Hence, the computational complexity of Algorithm 1 is $O\left(\epsilon_{n}\left|\gamma_{n}\right|\right)$.

The time needed by Algorithm 1 in order to converge to a stable solution at system initialization is limited by the maximum number of iterations, and can be computed as $\frac{\epsilon_{n}}{\Delta \epsilon} t_{i}$. A change of the error share of child $j$ initiated by parent node $n$ can be propagated in the whole subtree $T_{j}$ during the same protocol iteration, as valid rate estimates exist at all aggregating nodes in the subtree. Algorithm adaptation during system run-time is performed much faster, as presented next.

Observe that performing the optimization algorithms at all parent processes, starting at the root monitoring process with total error budget $\epsilon_{M}$ yields the local error shares at all processes, hence it offers a distributed, likely sub-optimal solution for the optimization problem presented in Section IV.
TABLE I

Simulation PARAMETERS

\begin{tabular}{c|c}
\hline Value set $S$ & {$[1, \ldots, 124]$} \\
\hline Estimation Window & $30 \mathrm{~s}$ \\
\hline Algorithm Iteration Window $t_{i}$ & $1 \mathrm{~s}$ \\
\hline Error Step $\Delta \epsilon$ & 2 \\
\hline Variable sampling rate & $1 \mathrm{~s}$ \\
\hline Rate threshold $\frac{\delta R}{R_{n}^{\hat{i n}}}$ & $20 \%$ \\
\hline Normalized Error Objective $\bar{\epsilon}$ & $\frac{D\left[H_{0}(t), H_{\text {real }}(t)\right]}{N}$ \\
\hline Protocol Initialization & $70 \mathrm{~s}$ \\
\hline Monitoring time & $100 \mathrm{~s}$ \\
\hline Error Computation Period & $0.5 \mathrm{~s}$
\end{tabular}

\section{E. Dynamic $\epsilon(\vec{n})$ Re-computation}

Algorithm 1 runs at system initialization and computes an appropriate error share that is propagated along the aggregation tree, starting from the root down. As the behavior of local values changes in time, we need to adapt the error share allocation in order to reflect the new system status. This implies the redistribution of the error budget among children at every parent process.

The dynamic re-computation of the error allocation, locally at each process $n$, is event-driven and requires rerunning Algorithm 1. Events are represented by (i) an increase of the parent error share $\epsilon_{n}$ by $\Delta \epsilon$, (ii) a decrease of $\epsilon_{n}$ by $\Delta \epsilon$, (iii) a local reallocation decision taken by the parent in case its current incoming rate estimate $\hat{R_{n}^{i n}}$ is larger by $\delta R$ than the original incoming rate at the time of the last error share allocation. $\delta R$ is a control parameter of our protocol. A larger value implies less frequent reallocations, and hence a more stable system at the expense of a larger protocol overhead.

In case of event (i), parent $n$ has enough information to reallocate the extra error share to its children by running an additional iteration of Algorithm 1 . However, for events (ii) and (iii), observe that $\sum_{j \in \gamma_{n}} \epsilon_{j} \geq \epsilon_{n}$ and a reallocation of the shares automatically implies the reduction of the share of at least one child.

In these cases we apply the concept of error share shrinking [3] which implies that we reduce the error share of all children by $\Delta \epsilon$ such that under the new error allocation $\sum_{j \in \gamma_{n}} \epsilon_{j} \leq \epsilon_{n}$. For the established time window $t_{i}$ we recompute the estimates $R_{j}^{\hat{o u t}}$ and we re-run Algorithm 1 starting with the new error allocation vector and the newly computed rate estimates for all children. We differentiate our method from [3] as we only apply the shrinking process locally, and event-based, e.g., only when needed. Hence, we expect our solution to be more efficient and more adaptive.

\section{Simulation Results}

We evaluate our proposed protocol through extensive simulations using the SIMPSON simulator [16]. We use five static network topologies containing $N=82,164,328,654$ and 1308 nodes respectively, generated by GoCast with a target connectivity of 5 . The aggregating trees built on top of these topologies have a maximum out-degree of 6 . The local management variable in the simulation experiments represents the number of HTTP flows traversing a given node. The monitored aggregate is the histogram of the variable in the 


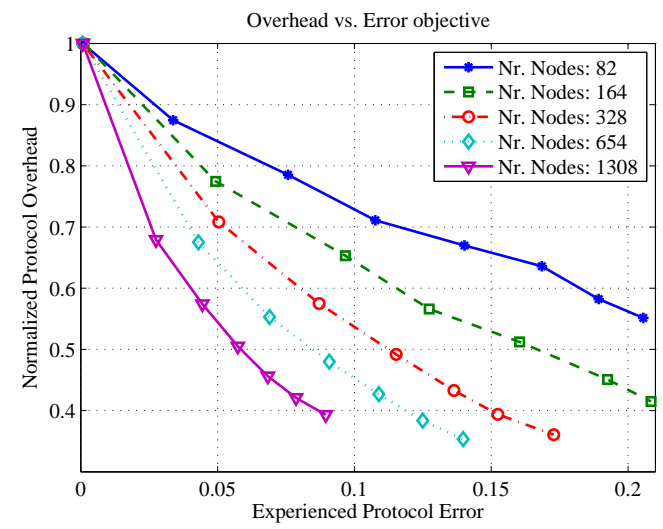

Fig. 2. Normalized protocol overhead vs. experienced error $\bar{\epsilon}$.

network. The local variables are updated asynchronously based on packet traces captured on two 1Gbit/s links that connect the University of Twente to a research network [17]. We use these traces in order to create events that are processed by the simulator. Each event changes the local variable of a node at a given time. All simulations start with a protocol initialization period during which our protocol constructs the aggregation tree, Algorithm 1 initializes, and each parent node computes an initial estimate $\hat{R_{n}^{i n}}$. Specific simulation parameters are presented in Table I.

We are first interested in the controllability of our protocol, and we test the reduction in protocol overhead when we increase the error objective for all simulation scenarios. We measure the total number of forwarded messages in the aggregation tree during the monitoring time. We do not count the leaf updates to their parents as they are not affected by the error objective (Section V). Figure 2 presents the obtained results normalized with the total number of messages exchanged for an error objective $\epsilon_{M}=0$. We observe a significant drop in total protocol overhead even for small error objectives. It is also noticeable that the performance of our protocol increases for larger networks, as these topologies offer more flexibility in error-share allocation.

Next, we test the scalability of HistAgg by computing the maximum link utilization in all network scenarios, for various error objectives. Link utilization is computed as the number of messages per second exchanged by our protocol on a given link, averaged over the monitoring time (Figure 3). We observe that even for large networks, a minimum increase in the error objective leads to a dramatic reduction in link utilization. For error objectives above $5 \%$ the maximum link utilization is approximately the same regardless of the network size. Such result has been expected as the protocol distributes the largest error-shares to nodes closer to the root where the link utilization is higher. At the same time, HistAgg always allocates an available error-share to the child that produces the maximum protocol overhead (hence the largest link utilization), trying to decrease it. These results prove that our protocol scales well with increased network size which, together with its low computational complexity, makes it a suitable candidate for the monitoring of large-scale networks.

Finally we are interested in comparing the accuracy of the

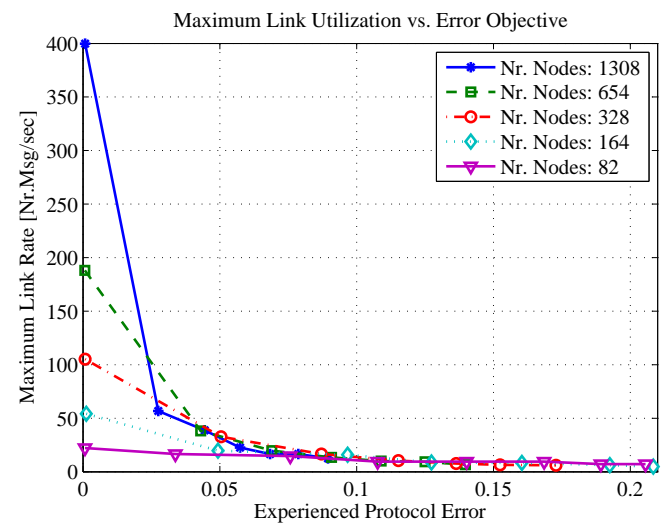

Fig. 3. Maximum link utilization vs. experienced error $\bar{\epsilon}$.

protocol's error objective compared to the real error in the obtained results. With this respect, we test our protocol for various pre-set error objectives and we compute the actual aggregation error at the root node. The aggregation error is computed periodically as the distance between the current aggregated histogram available at the root node, and the real histogram of the local variables computed from the available traces. Figure 4 presents the results for one network scenario, while observed results for all other scenarios are similar. We observe a small under-valuation of the total error for extremely low error objectives, while the real error tends to be smaller than the pre-set error objectives for higher values. This is explained by the fact that for higher error-objectives we observe the cancelling effect of histogram aggregation at intermediate nodes. Parents wait for more child updates before forwarding the aggregate up the tree, hence increasing the chance of observing updates with changes that cancel each other out. This effect cannot be observed in asynchronous systems at low error objectives, as intermediate nodes must forward almost all incoming updates without waiting for more updates that might cancel some of the changes. While this behavior is positive for the performance of our protocol, we do not have a formal model to quantify or predict the difference between the pre-set error objective, and the real experienced error. We plan to investigate this aspect in the future.

\section{DISCUSSION}

HistAgg represents our distributed solution for histogram aggregation over aggreagtion trees with an accuracy objective. We define the objective as the maximum tolerated distance between the aggregated histogram and the real variable value distribution in the system. As Hist Agg is used as a means to provide the management station with information on which it can apply various management functions, our error objective should be reflected on a function-dependent error metric. With this respect, specific error objectives for various management functions should be defined, mapped and evaluated against our defined $D$ metric. Such analysis is omitted in this paper, and is left for future work.

In the presented optimization problems we are concerned with the minimization of protocol overhead (computed as the 


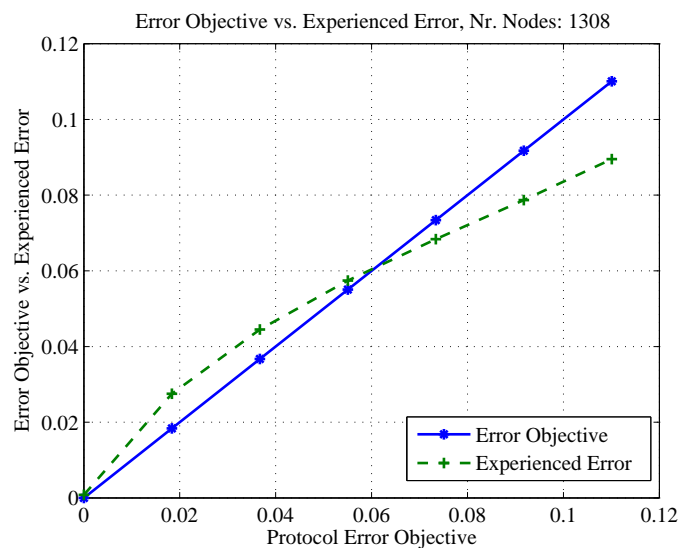

Fig. 4. Error objective $\overline{\epsilon_{M}}$ vs. experienced error $\bar{\epsilon}$.

rate of sent messages) needed in order to aggregate the partial value vectors. Observe that our protocol can be straightforwardly adapted in order to minimize the total processing load at system nodes, as computational resources spending is eventbased, i.e., a node uses computational resources only when a new message update is received.

While in HistAgg we are interested in minimizing the total system resource consumption, achieving fairness among system nodes is a worthy goal in resource limited systems, e.g., sensor networks. With this respect, our protocol can straightforwardly be adapted to take into account a maximum load on any system node/link as an additional constraint. While our optimization goal still remains the minimization of the global overhead, and does not ensure complete fairness among nodes, such a constraint limits our solution space to more fair allocations. This would satisfy more general system constraints, e.g., the minimum lifetime of a network. We have already taken a step towards achieving this goal in Algorithm 1 where we always allocate a new error share to the child process that induces the largest link overhead.

Finally, we notice that our analysis and solution take into account only integer-valued local variables with a finite value space $S$. Our solution can be extended to the monitoring of real-valued variables by incorporating an initial quantization step. Local values could be quantized with quantization step $Q$. The size of $Q$ determines the size of the aggregation vector $H$, and hence, the size of the exchanged messages in the system. In this case, our goal would be the minimization of the protocol overhead computed as number of bytes exchanged. Our optimization problem translates then into optimizing the split of the error objective $\epsilon_{M}$ into a quantization error $\epsilon_{Q}$ (which influences the quantization step $Q$ and the size of messages), and an update error $\epsilon_{\tau}$ (which influences the rate of updates propagated up the trees). The investigation of this problem is left to future work.

\section{CONCLUSIONS}

In this paper we present a distributed protocol for the aggregation of the histogram of a monitored local variable in large scale systems. Our protocol provides the management station with timely information about the distribution of the values of a monitored variable with minimal protocol overhead, while satisfying a total system accuracy objective. Our solution can control its operation point on the trade-off curve between protocol overhead and system accuracy objective. Based on the aggregated histogram, the management station has the flexibility to perform various statistical functions either sequentially, or in parallel. This is not possible with traditional aggregation mechanisms based on pushing the aggregation function inside the network. To the best of our knowledge, our work is the first that offers a detailed analysis of the problem of distributed histogram aggregation over aggregation trees. We include a complete global optimization problem formulation and formalization, and a full-fledged distributed solution containing heuristic tree-based algorithms suitable for real system implementations. Our results emphasize the tradeoff between the accuracy objective and protocol overhead, and suggest that the protocol efficiency increases with system size.

\section{REFERENCES}

[1] A. Gonzalez and R. Stadler, "A-GAP: An Adaptive Protocol for Continuous Network Monitoring with Accuracy Objectives," IEEE Transactions on Network and Service Management, 2007.

[2] G. Cormode, M. Garofalakis, S. Muthukrishnan, and R. Rastogi, "Holistic Aggregates in a Networked World: Distributed Tracking of Approximate Quantiles," in Proceedings of ACM SIGGMOD, June 2005.

[3] A. Deligiannakis, Y. Kotidis, and N. RoussoPoulos, "Hierarchical InNetwork Data Aggregation with Quality Guarantees," in Proceedings of EDBT Crete, Greece, March 2004.

[4] A. Boulis, S. Ganeriwal, and M. B. Srivastava, "Aggregation in Sensor Networks: An Energy - Accuracy Trade-off," Elsevier Ad-Hoc Networks Journal (special issue on sensor network protocols and applications), pp. 317-331, 2003.

[5] B. Krishnamaachari, D. Estrin, and S. Wicker, "The Impact of Data Aggregation in Wireless Sensor Networks," in Proceedings of the International Workshop of Distributed Event-Based Systems, Vienna, Austria, July 2002.

[6] M. A. Sharaf, J. Beaver, A. Labrinidis, and P. K. Chrysantis, "Balancing Energy Efficiency and Quality of Aggregate Data in Sensor Networks," ACM International Journal on Very Large Data Bases, vol. 13, no. 4, pp. 384-403, December 2004.

[7] C. Olson, J. Jiang, and J. Widom, "Adaptive Filters for Continuous Queries over Distributed Data Streams," in Proceedings of ACM SIGGMOD, San Diego, USA, June 2003.

[8] S.-H. Cha and S. N. Srihari, "On Measuring the distance between Histograms," Elsevier, The Journal of Pattern Recognition, vol. 35, pp. 1355-1370, 2002.

[9] A. C. Gilbert, Y. Kotidis, S. Muthukrishnan, and M. J. Strauss, "How to Summarize the Universe: Dynamic Maintainance of Quantiles," in Proceedings of the 28th VLDB Conference, 2005.

[10] M. Greenwald and S. Khanna, "Space-Efficient Online Computation of Quantile Summaries," in Proceedings of ACM SIGGMOD, May 2001.

[11] Y. Rubner, C. Tomasi, and L. J. Guibas, "A Metric for Distributions with Applications to Image Databases," in Proceedings of the 1998 IEEE International Conference on Computer Vision, 1998.

[12] J. Wang, "Algorithms for New Distance Measures between Histograms," in Proceedings of IEEE, 2001.

[13] N. Thaper and S. Guha, "Dynamic Multidimensional Histograms," in Proceedings of ACM SIGGMOD, June 2002.

[14] M. Dam and R. Stadler, "A Generic Protocol for Network State Aggregation," in Proceedings of Radiovetenskap och Kommunication (RVK), Linkoping, Sweden, June 2005.

[15] IEEE, “ANSI/IEEE Std 802.1D,” IEEE, 1998 Edition.

[16] K. Lim and R.Stadler, "SIMPSON - a SIMple Pattern Simulator fOr Networks," http://www.s3.kth.se/lcn/software/simpson.shtml, July 2006.

[17] R. van der Meent and A. Pras, "Traffic Measurement Data Repository, University of Twente," http://traffic-repository.ewi.utwente.nl, May 2006. 